\title{
Development of CNS metastases in breast cancer patients treated with curative intent: a case-control study
}

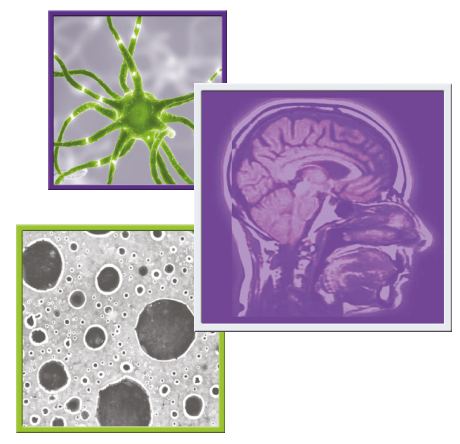

\author{
Katherine Chakrabarti ${ }^{\ddagger 1}$, Leigh K Swartz ${ }^{\ddagger 1}$ (D) Anoop Gill1, Fang Fang ${ }^{2}$, Kelley M \\ Kidwell ${ }^{2}$ iD \& Aki Morikawa*,1 (iD \\ ${ }^{1}$ Department of Internal Medicine, University of Michigan Health System, 1500 E. Medical Center Drive, Ann Arbor, MI 48109, USA \\ ${ }^{2}$ Department of Biostatistics, University of Michigan, School of Public Health, 1415 Washington Heights, Ann Arbor, Ml 48109, \\ USA \\ *Author for correspondence: morikawa@med.umich.edu \\ ${ }_{\ddagger}^{\ddagger}$ Authors contributed equally
}

Background: The aim of this study was to identify risk factors that may predispose breast cancer patients to the development of CNS metastases. Materials \& methods: We conducted a matched case-control study of breast cancer patients treated with surgery with curative intent. A total of 71 cases and 71 controls were analyzed, matched by year of surgery. Results: In our multivariable model, positive lymph node status (odds ratio [OR]: 5.08; Cl: 2.04-12.65), the use of neoadjuvant chemotherapy (OR: 6.02; Cl: 2.06-17.57) and triple-negative breast cancer (OR: $5.44 ; \mathrm{Cl}: 1.99-14.90)$ were statistically significant predictors of the development of CNS metastases. Conclusion: Women with certain risk factors have an increased odds of developing CNS metastases and evaluation of utility in brain metastases screening should be considered.

First draft submitted: 4 May 2020; Accepted for publication: 29 June 2020; Published online: 18 September 2020

Keywords: breast cancer $\bullet$ breast cancer brain metastases $(B C B M) \bullet C N S \bullet C N S$ metastases $\bullet$ estrogen receptor (ER) - HER2 • triple-negative breast cancer

Breast cancer is the most common malignancy in women, affecting one in eight women in the USA during their lifetime [1]. Of the complications arising from the disease, metastases to the CNS are one of the most feared among both clinicians and patients [2-4]. CNS metastases can occur as multiple lesions or solitary lesions and can affect any of the CNS related tissues including the dura, brain parenchyma or leptomeninges. Median overall survival was 13.8 months in a large study of breast cancer patients with newly diagnosed CNS metastases [5]. Among patients diagnosed and treated for early stage breast cancer, approximately 3-6\% will go on to develop CNS metastases while an estimated $10-16 \%$ of patients with stage IV disease at diagnosis develop CNS metastases [4]. In HER2-positive patients, approximately $9 \%$ have CNS metastases at diagnosis and another $22 \%$ will later develop CNS metastases, as demonstrated in a prospective, observational study [6]. Currently there is an evidence to suggest that the incidence of breast cancer brain metastases (BCBM) is increasing [7,8]. This has been hypothesized to be due to improved imaging diagnostics and increased overall breast cancer survival through advanced multimodality treatment approaches [7]. Ongoing research is focused on identifying high-risk breast cancer populations in terms of tumor genomics and biology, and assessing the need for screening brain imaging strategies in high-risk groups.

The diagnosis and treatment of CNS metastases represents an unmet need in the field of breast oncology. In the breast cancer population, practice guidelines do not recommend any routine screening for CNS metastases in asymptomatic patients for any stage or breast cancer subtype; therefore, diagnosis of the disease usually occurs after the development of neurologic symptoms [9,10]. A recent retrospective review of 100 patients with HER2-positive breast cancer demonstrated that diagnosing BCBM when patients were asymptomatic was associated with decreased use of whole brain radiation therapy, fewer brain lesions and increased overall survival [11]. In the Systemic Therapies for HER2-positive Metastatic Breast Cancer Study, patients with CNS metastases had lower quality of life scores than those who did not have CNS metastases at diagnosis [6]. 
Additionally, outcomes in patients with BCBM are worse as compared with patients with brain metastases from non-small-cell lung cancer (NSCLC) where screening for brain metastases is routinely recommended in patients at diagnosis with stage II disease or higher [12,13]. It is estimated that up to $10 \%$ of NSCLC patients have brain metastases at the time of diagnosis and $20-40 \%$ will later develop brain metastases [14]. In a recent retrospective review comparing patients with NSCLC and breast cancer, the authors concluded that breast cancer patients were more likely to have seizures, leptomeningeal disease, neurologic symptoms and brainstem involvement at the time of diagnosis of brain metastases, in addition to being more likely to receive upfront whole brain radiation therapy and die from neurologic causes [13]. Progression of disease and development of brain metastases are well recognized to lead to worse quality of life.

Given the poor prognosis associated with the development of CNS metastases, over the past two decades there has been a movement to identify patients who will go on to develop this complication [15]. A number of disease and patient-specific factors have been suggested to portend an increased risk of development of CNS metastases. Studies have reported hormone receptor negativity, HER2 receptor positivity, younger age at diagnosis, presence of $B R C A$ mutation and a more advanced stage and grade at diagnosis to be associated with the risk of CNS metastases [16-20]. Despite multiple studies, the best strategy to risk stratify breast cancer patients for the development of CNS metastases remains unclear. It is clear that the population that will develop CNS metastases is markedly heterogeneous, making prognostication of disease progression and survival difficult. We analyzed a contemporary cohort of patients who were treated with curative intent for development of CNS metastases. The aim of this retrospective study was to identify clinical and histopathologic characteristics of women with breast cancer who have increased odds of developing CNS metastases.

\section{Patients \& methods}

We conducted a matched case-control study of patients with breast cancer treated with surgery with a curative intent between January 2000 and 2015 at the University of Michigan Hospital in Ann Arbor, Michigan (USA). Cases were defined as women $\geq 18$ years of age with breast cancer who underwent definitive surgery with curative intent, which included primary or secondary surgery for positive margins, who then went on to develop CNS metastases during the follow-up period. Subjects were excluded if metastatic disease was present at diagnosis or if they had a second primary malignancy with potential to metastasize to the CNS. Controls were defined as women $\geq 18$ years of age with breast cancer who underwent definitive surgery with curative intent who did not develop CNS metastases in the study period. Cases and controls were matched one to one by year of surgery.

Clinicopathologic information was collected by retrospective chart review, including patient demographics; menopausal status; dates of diagnosis of breast cancer, subsequent brain metastases and other metastases; treatments. including neoadjuvant and adjuvant chemotherapy, hormone therapy and radiation and clinical outcomes. The institutional review board approved this study.

\section{Statistical analysis}

Associations of demographic, clinicopathologic and treatment variables with development of CNS metastases were first assessed individually in an unconditional logistic model controlling for year of diagnosis. Although this is a matched case-control study, an unconditional logistic model was used for gains in precision considering the small sample size [21]. Considering uncorrelated variables, backward elimination was used to fit a multivariable model keeping variables with $\mathrm{p}<0.1$ including age, race, pathological stage, lymph nodes status, triple-negative status (ER, PR, HER2 all negative) and neoadjuvant chemotherapy receipt.

\section{Results}

From the period of 2000-2015, we identified 71 sequential patients with definitive treatment for nonmetastatic breast cancer who went on to develop CNS metastases and matched 71 controls. The median time from diagnosis of breast cancer to development of CNS metastases was 27.36 months. The median follow-up period (time of breast cancer diagnosis to death or last follow-up) for cases and controls combined was 68.46 months (range: 2.08-203.70 months). Descriptive statistics are given in Table 1.

There are no guidelines for screening for CNS metastases in patients with breast cancer, so as expected, the majority of our patients were diagnosed once they developed symptoms. In total, 65 patients $(91.15 \%)$ developed symptoms prompting brain imaging $(64 / 71,90.14 \%)$ or lumbar puncture $(1 / 71,1.41 \%)$ resulting in the diagnosis 


\section{Table 1. Demographic characteristics.}

\section{Characteristics}

Age (years), mean (SD)

Race:

- Caucasian

African-American or Black

- Other

- Unknown

Cancer type:

- Ductal

- Other

Clinical stage:

- Stage I

- Stage II

- Stage III

- Unknown

Pathological stage:

- Stage I

\section{- Stage II}

- Stage III

- Unknown

Lymph node status:

- Negative

- Positive

- Unknown

Receptor status:

- ER-positive

- PR-positive

- HER2-positive

Menopause status:

- Premenopausal

- Postmenopausal

- Perimenopausal

- Unknown

Primary surgery:

- Lumpectomy

- Mastectomy

- Unknown

Axillary surgery:

- Sentinel LN biopsy

- Axillary LN dissection

- None

-U nknown

Treatment:

- Neoadjuvant chemotherapy

- Adjuvant chemotherapy

- Radiation

- Hormone therapy

ER: Estrogen receptor; LN: Lymph node; PR: Progesterone receptor; SD: Standard deviation.
Case $(n=71), n(\%)$

50.6 (13.72)

$58(81.7 \%)$

$10(14.1 \%)$

$1(1.4 \%)$

$1(1.4 \%)$

$1(1.4 \%)$

$62(87.3 \%)$

$9(12.7 \%)$

$12(16.9 \%)$

$30(42.3 \%)$

$28(39.4 \%)$

$1(1.4 \%)$

$4(5.6 \%)$

$20(28.2 \%)$

$44(62.0 \%)$

$3(4.2 \%)$

$30(42.3 \%)$

$41(57.7 \%)$

$0(0.0 \%)$

$29(40.8 \%)$

$20(28.2 \%)$

$22(31.0 \%)$

$36(50.7 \%)$

$33(46.5 \%)$

$0(0 \%)$

$2(2.8 \%)$

$31(43.7 \%)$

$40(56.3 \%)$

$0(0 \%)$

$30(42.3 \%)$

$36(50.7 \%)$

$2(2.8 \%)$

$3(4.2 \%)$

35 (49.3\%)

$36(50.7 \%)$

$50(70.4 \%)$

$27(38.0 \%)$
Control $(n=71), n(\%)$

$56.2(12.30)$

$61(85.9 \%)$

$2(2.8 \%)$

$6(8.5 \%)$

$1(1.4 \%)$

$1(1.4 \%)$

$62(87.3 \%)$

$9(12.7 \%)$

$46(64.8 \%)$

$17(23.9 \%)$

$8(11.3 \%)$

$0(0.0 \%)$

$13(18.3 \%)$

$35(49.3 \%)$

$20(28.2 \%)$

$3(4.2 \%)$

$58(81.7 \%)$

$12(16.9 \%)$

$1(1.4 \%)$

$53(74.6 \%)$

$44(62.0 \%)$

$19(26.8 \%)$

$16(22.5 \%)$

$36(50.7 \%)$

$1(1.4 \%)$

$18(25.4 \%)$

$45(63.4 \%)$

$25(35.2 \%)$

$1(1.4 \%)$

$52(73.2 \%)$

$17(23.9 \%)$

$0(0 \%)$

$2(2.8 \%)$

$6(8.5 \%)$

$27(38.0 \%)$

$40(56.3 \%)$

$46(64.8 \%)$ 


\begin{tabular}{|c|c|c|}
\hline Characteristics & Odds ratio $(95 \% \mathrm{Cl})$ & p-value \\
\hline Age (years) & $0.97(0.942-0.993)$ & 0.013 \\
\hline Race: & & 0.066 \\
\hline - Caucasian vs other & $0.95(0.058-15.484)$ & 0.968 \\
\hline - African-American or Black vs other & $5.01(0.212-118.260)$ & 0.318 \\
\hline - Asian vs other & $0.16(0.005-5.383)$ & 0.310 \\
\hline \multicolumn{3}{|l|}{ Cancer type: } \\
\hline - Ductal vs other & $1.00(0.371-2.692)$ & 1.000 \\
\hline Clinical stage: & & $<0.001$ \\
\hline - Stage II vs stage I & $7.27(2.988-17.680)$ & $<0.001$ \\
\hline - Stage III vs stage I & $15.52(5.393-44.671)$ & $<0.001$ \\
\hline Pathological stage: & & $<0.001$ \\
\hline - Stage II vs stage I & $1.81(0.518-6.340)$ & 0.352 \\
\hline - Stage III vs stage I & $7.68(2.192-26.923)$ & 0.001 \\
\hline \multicolumn{3}{|l|}{ Lymph node status: } \\
\hline - Positive vs negative & $6.69(3.053-14.650)$ & $<0.001$ \\
\hline \multicolumn{3}{|l|}{ Receptor status: } \\
\hline - ER-positive vs negative & $0.23(0.114-0.478)$ & $<0.001$ \\
\hline - PR-positive vs negative & $0.24(0.118-0.485)$ & $<0.001$ \\
\hline - HER2-positive vs negative & $1.17(0.561-2.444)$ & $<0.675$ \\
\hline \multicolumn{3}{|l|}{ Menopause status: } \\
\hline - Postmenopausal vs premenopausal & $0.41(0.193-0.873)$ & 0.021 \\
\hline \multicolumn{3}{|l|}{ Primary surgery: } \\
\hline - Mastectomy vs lumpectomy & $2.32(1.179-4.583)$ & 0.015 \\
\hline \multicolumn{3}{|l|}{ Axillary surgery: } \\
\hline - Sentinel LN biopsy vs axillary LN dissection & $0.27(0.127-0.557)$ & $<0.001$ \\
\hline \multicolumn{3}{|l|}{ Treatment: } \\
\hline - Neoadjuvant chemo: yes vs no & $10.96(4.162-28.864)$ & $<0.001$ \\
\hline - Adjuvant chemo: yes vs no & $1.64(0.839-3.216)$ & 0.148 \\
\hline - Radiation: yes vs no & $1.88(0.931-3.799)$ & 0.078 \\
\hline - Hormone therapy: yes vs no & $0.33(0.165-0.653)$ & 0.002 \\
\hline
\end{tabular}

of CNS metastases. A small number $(4 / 64,5.63 \%)$ were diagnosed based on incidental imaging findings. Two patients $(2.82 \%)$ had missing data and were diagnosed in an unknown manner.

The mean age overall was 53.4 years with cases significantly younger than controls ( 50.6 vs 56.2 years; p-value 0.013 ) and more likely to be premenopausal (50.7 vs $22.5 \%$; p-value 0.021$)$. There were no significant racial differences between the two groups. More cases had clinical stage II disease or higher and pathologic stage III or higher $(\mathrm{p} \leq 0.001)$. Results of univariate analysis are given in Table 2.

Cases were more likely than controls to have positive lymph nodes $(57.5$ vs $16.9 \%$; $p<0.001$ ) and undergo axillary lymph node dissection $(50.7$ vs $23.9 \%$; $p<0.001)$. Regarding treatment, cases were more likely to receive neoadjuvant chemotherapy, mastectomy and axillary lymph node dissection. The most common chemotherapy drugs used in our study were doxorubicin, cyclophosphamide and paclitaxel. For neoadjuvant chemotherapy, 100\% of the controls and $88.58 \%$ of cases had a taxane-based regimen. For adjuvant chemotherapy, $58.33 \%$ of the cases and $59.26 \%$ of the controls had a taxane-based regimen. Conversely, cases were less likely than controls to be ER-positive ( 40.8 vs $74.8 \%$; $p<0.001)$ or PR-positive $(28.2$ vs $62.0 \%$; $p<0.001)$ and therefore, less likely to receive hormone therapy ( 38.0 vs $64.8 \%, \mathrm{p}=0.002)$. HER2-positive status was not significantly associated with odds of brain metastases. Although cases were numerically more likely to receive radiation than controls ( $70.4 \mathrm{vs}$ $56.3 \%)$, this was not statistically significant $(\mathrm{p}=0.078)$. 


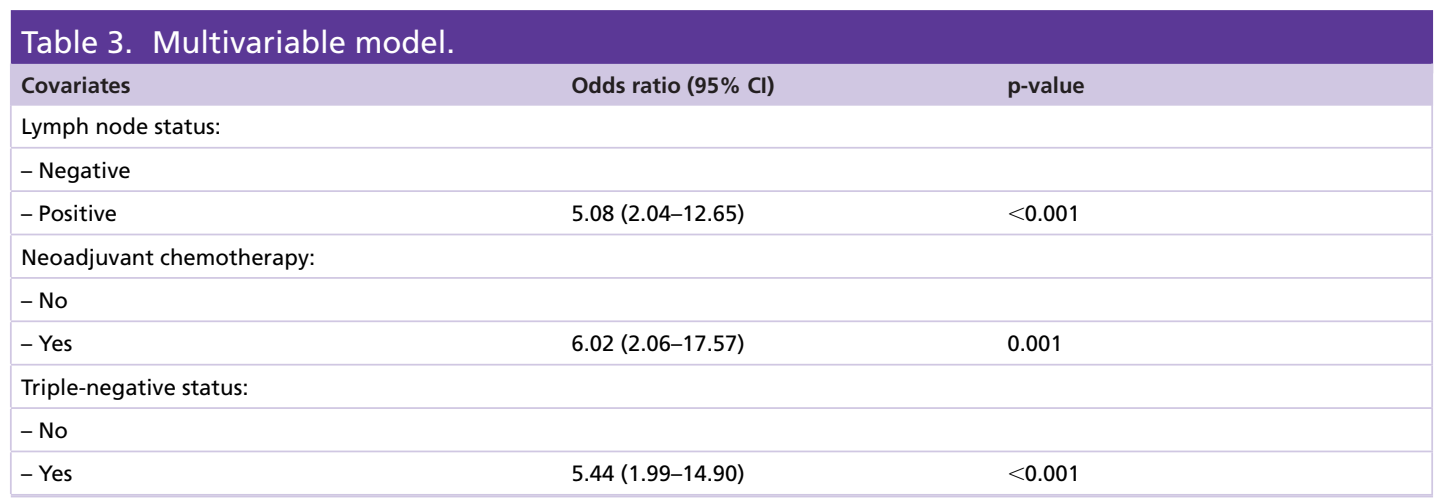

In our final multivariable model, positive lymph node status (OR: 5.08; CI: 2.04-12.65), the use of neoadjuvant chemotherapy (OR: 6.02; CI 2.06-17.57) and triple-negative breast cancer (OR: 5.44; CI: 1.99-14.90) were significant predictors of the development of CNS metastases (all $\mathrm{p} \leq 0.001$ ). Results are presented in Table 3 .

\section{Discussion}

The purpose of this study was to identify risk factors that may predispose breast cancer patients treated in the curative setting to the development of CNS metastases. Identification of high-risk patients may facilitate earlier detection of BCBM, and may influence current screening guidelines in select populations.

Through a retrospective, case-control analysis, we identified demographic variables, tumor characteristics and treatment regimens of interest. We identified triple-negative status, lymph node positivity and receipt of neoadjuvant chemotherapy as independent risk factors for the development of CNS metastases. Our analysis demonstrates that higher stage disease at presentation and thus, the use of more aggressive upfront treatment is associated with increased odds of developing CNS metastases. In univariate analysis, younger age and premenopausal status at diagnosis were also associated with increased odds of developing CNS metastases.

Our study is consistent with prior analyses that have demonstrated hormone receptor negativity, younger age at diagnosis and more advanced stage at diagnosis as risk factors for the development of CNS metastases. Tham et al. found that younger patients with hormone receptor-negative, highly proliferative, genomically unstable and p53-altered tumors were at increased relative risk for CNS metastases, while HER2 expression and adjuvant systemic therapies did not increase this risk [16]. Pestalozzi et al. found a high incidence of brain metastases among patients with age $<35$ years, tumor grade 3, $>4$ positive nodes, tumor size $>2 \mathrm{~cm}$ and ER-negative disease [17]. Nam et al. found that triple-negative and HER2-positive/ER-negative tumors had a high-risk of metastatic CNS disease [18]. Azim et al. demonstrated that HER2 positivity, large tumors and axillary lymph node metastases predicted development of brain metastases in patients presenting with locoregional disease, while HER2 positivity, presence of lung metastasis and shorter relapse-free interval were predictive of developing brain metastases in patients with metastatic breast cancer at presentation [20].

Interestingly, a recent case-control study observed that $B R C A$-mutated breast cancer patients are more likely to develop brain metastases in a short time interval compared with wild-type $B R C A$ controls matched for age, stage, ER expression and HER2 expression [19]. While, our study did not investigate germline genetic mutations in our cohort, younger patients with triple-negative breast cancer are more likely to have genetic mutations such as BRCA1. This study suggests that further investigation aimed at identifying germline genetic mutations may be beneficial to identifying additional risk factors for development of CNS metastases. Additionally, analysis of genomic and molecular information from primary tumors may help to identify somatic mutations that would predispose a patient to developing CNS metastases.

Contrary to prior studies, our analysis did not identify HER2 positivity as a risk factor [20,22-24]. Undoubtedly, outcomes in patients with HER2-positive disease are improving in the era of HER2-targeted therapy. It is possible the effect of HER2 was not seen due to the small sample size of this case-control study, wherein only 22 cases (31\%) and 19 controls (26.8\%) were HER2-positive. Though not statistically significant, there was a slight trend toward cases being HER2-positive. It is also possible that HER2 positivity is a more important risk factor for developing BCBM in the metastatic setting, as opposed to the early stage setting studied in this analysis [16,17]. Our data are interestingly consistent with an observation of lower brain metastasis incidence reported in the 
KATHERINE trial, an adjuvant trial conducted in high-risk HER2-positive breast cancer patients [25]. This study shows a $4.3 \%$ incidence among high-risk early stage HER2-positive patients in the trastuzumab arm compared with a 7.5\% incidence reported in early stage triple-negative breast cancer patients from a single-institution study [26]. Additionally, we included patients with leptomeningeal disease only, although the number of such patients was small in our study population which may further explain why our study did not identify HER2 positivity as a risk factor. While HER2 positivity is an established risk factor for developing brain metastases in the metastatic setting, our result adds to the literature as our data suggest further need to evaluate HER2 subtype as a risk factor for brain metastasis development for patients treated in the curative setting with routine adjuvant anti-HER2 therapy. Our study has a number of limitations, including small sample size and retrospective nature of the analysis. Small sample size may have affected the nature of our conclusions, including lack of statistical significance for HER2 positivity. Brain metastases is a relatively low incident site of relapse in early breast cancer patients; therefore, our number was also limited by its epidemiology.

\section{Conclusion}

The identification of risk factors for the development of CNS metastases would allow oncologists to re-examine screening in the high-risk, target population and design prospective screening trials. Our case-control study identified three independent factors by multivariable analysis associated with increased odds of developing CNS metastases, including triple-negative status, lymph node positivity and prior receipt of neoadjuvant chemotherapy. Future efforts are being considered to additionally focus on identifying genomic or molecular information that may add further clarification to risk stratification in our study population. Further study is needed in order to rethink guidelines for identification of asymptomatic brain metastases in high-risk patients. If CNS metastases were to be diagnosed earlier in the disease course when a patient is more likely to have limited disease burden and a better functional status, this may then allow for earlier initiation of treatment, potential use of additional therapy options, introduction of secondary prevention, improved survival and better quality of life.

\section{Future perspective}

As continued research elaborates the risk factors for development of CNS metastases, consensus screening guidelines may evolve within the coming years to incorporate neuroimaging at diagnosis in high-risk populations. Additionally, it will become increasingly more common to sequence breast cancer tissues samples which may lead to further identification of genetic risk factors for development of CNS metastases. The pathway forward involves creating a more personalized approach to cancer care.

\section{Summary points}

- Routine screening for CNS metastases is not currently recommended in asymptomatic patients with breast cancer.

- Most breast cancer patients with CNS metastases are diagnosed when they present with neurologic symptoms.

- While breast cancer is one of the solid tumor types that is more likely metastasize to the CNS, the actual risk of developing CNS metastases is still low among early stage breast cancer patients who are treated initially with curative intent.

- There is a need to identify high-risk breast cancer populations in terms of tumor genomics and biology, and to assess the need for screening brain imaging strategies in high-risk groups.

- Positive lymph node status, the use of neoadjuvant chemotherapy and triple-negative breast cancer were statistically significant predictors of the development of CNS metastases.

- Prospective evaluation of CNS metastasis screening in breast cancer patients is warranted and may be refined by a risk stratification approach.

- Further study is needed in order to rethink guidelines for identification of asymptomatic brain metastases in high-risk patients.

\footnotetext{
Author contributions

K Chakrabarti contributed substantially to the design of the study, analysis of the data and drafting of the manuscript. LK Swartz contributed substantially to the analysis of the data and was a major contributor in writing and revising the manuscript. A Gill collected the initial data, maintained the database used for the study and pulled all necessary data used in the manuscript. F Fang statistically analyzed and interpreted the data, and created tables used in the manuscript. KM Kidwell statistically analyzed and interpreted the data, and created tables used in the manuscript. A Morikawa created the concept for the study, designed the study, analyzed the data and was a major contributor in writing and revising the manuscript. A Morikawa is the principal investigator for the study. All authors read and approved the final manuscript.
} 
Financial \& competing interests disclosure

The Conquer Cancer Foundation (CCF) of American Society of Clinical Oncology (ASCO): Predicting brain metastases in breast cancer patients through molecular profiling: 16-PAF01301. The authors declare that they have no competing interest. The authors have no other relevant affiliations or financial involvement with any organization or entity with a financial interest in or financial conflict with the subject matter or materials discussed in the manuscript apart from those disclosed.

No writing assistance was utilized in the production of this manuscript.

\section{Ethical conduct of research}

The authors state that they have obtained appropriate institutional review board approval from the University of Michigan Institutional Review Board (Study ID: HUM00108319) and have followed the principles outlined in the Declaration of Helsinki for all human experimental investigations. A waiver of informed consent was obtained.

\section{Open access}

This work is licensed under the Attribution-NonCommercial-NoDerivatives 4.0 Unported License. To view a copy of this license, visit http://creativecommons.org/licenses/by-nc-nd/4.0/

\section{References}

Papers of special note have been highlighted as: $\bullet$ of interest; $\bullet \bullet$ of considerable interest

1. McPherson K, Steel CM, Dixon JM. Breast cancer-epidemiology, risk factors, and genetics. BMJ 321(7261), 624-628 (2000).

2. Pestalozzi BC. Brain metastases and subtypes of breast cancer. Ann. Oncol. 20(5), 803-805 (2009).

3. Martin AM, Cagney DN, Catalano PJ. Brain metastases in newly diagnosed breast cancer: a population-based study. JAMA Oncol. 3(8), 1069-1077 (2017).

- Identifies the incidence proportion and median survival of patients with breast cancer brain metastases using the SEER database.

4. Lin NU, Bellon JR, Winer EP. CNS metastases in breast cancer. J. Clin. Oncol. 22(17), 3608-3617 (2004).

5. Sperduto PW, Kased N, Roberge D et al. Effect of tumor subtype on survival and the graded prognostic assessment for patients with breast cancer and brain metastases. Int. J. Radiat. Oncol. Biol. Phys. 82(5), 2111-2117 (2012).

- Refines the breast-graded prognostic assessment, a tool used to estimate prognosis of subtypes of patients with breast cancer brain metastases.

6. Hurvitz SA, O'Shaughnessy J, Mason G et al. Central nervous system metastasis in patients with HER2-positive metastatic breast cancer: patient characteristics, treatment, and survival from SystHERs. Clin. Cancer Res. 25(8), 2433-2441 (2019).

•• In Systemic Therapies for HER2-positive Metastatic Breast Cancer Study, a prospective, observational study, HER2-positive patients were followed for the development of CNS metastases. Patients with CNS metastases had lower quality of life scores than those who did not have CNS metastases at diagnosis.

7. Kotecki N, Lefranc F, Devriendt D, Awada A. Therapy of breast cancer brain metastases: challenges, emerging treatments and perspectives. Ther. Adv. Med. Oncol. 10, 1758835918780312 (2018).

8. Nieder C, Spanne O, Mehta MP, Grosu AL, Geinitz H. Presentation, patterns of care, and survival in patients with brain metastases: what has changed in the last 20 years? Cancer 117(11), 2505-2512 (2011).

9. Bowman KM, Kumthekar P. Medical management of brain metastases and leptomeningeal disease in patients with breast carcinoma. Future Oncol. 14(4), 391-407 (2018).

10. National Comprehensive Cancer Network. Breast cancer version 1.2019 (2019). www.nccn.org/professionals/physician_gls/pdf/breast.pdf

11. Morikawa A, Wang R, Patil S et al. Characteristics and prognostic factors for patients with HER2-overexpressing breast cancer and brain metastases in the era of HER2-targeted therapy: an argument for earlier detection. Clin. Breast Cancer. 18(5), 353-361 (2018).

12. National Comprehensive Cancer Network. Non-small cell lung cancer version 1.2019 (2018). www.nccn.org/professionals/physician_gls/pdf/nscl.pdf

13. Cagney DN, Martin AM, Catalano PJ. Implications of screening for brain metastases in patients with breast cancer and non-small cell lung cancer. JAMA Oncol. 4(7), 1001-1003 (2018).

14. Ali A, Goffin JR, Arnold A, Ellis PM. Survival of patients with non-small-cell lung cancer after a diagnosis of brain metastases. Curr. Oncol. 20(4), e300-e306 (2013).

15. Graesslin O, Abdulkarim BS, Coutant C et al. Nomogram to predict subsequent brain metastasis in patients with metastatic breast cancer. J. Clin. Oncol. 28(12), 2032-2037 (2010).

16. Tham Y-L, Sexton K, Kramer R, Hilsenbeck S, Elledge R. Primary breast cancer phenotypes associated with propensity for central nervous system metastases. Cancer 107(4), 696-704 (2006). 
- Identifies increased relative risk for CNS metastases in younger patients with hormone receptor-negative, highly proliferative, genomically unstable and p53-altered tumors. HER2 expression and adjuvant systemic therapies did not increase this risk.

17. Pestalozzi BC, Zahrieh D, Price $\mathrm{KN}$ et al. Identifying breast cancer patients at risk for central nervous system (CNS) metastases in trials of the International Breast Cancer Study Group (IBCSG). Ann. Oncol. 17(6), 935-944 (2006).

- Finds a high incidence of brain metastases among patients with age $<35$ years, tumor grade 3, $>4$ positive nodes, tumor size $>2 \mathrm{~cm}$ and ER-negative disease.

18. Nam B-H, Kim SY, Han H-S et al. Breast cancer subtypes and survival in patients with brain metastases. Breast Cancer Res. 10(1), R20 (2008).

19. Zavitsanos PJ, Wazer DE, Hepel JT, Wang Y, Singh K, Leonard KL. BRCA1 mutations associated with increased risk of brain metastases in breast cancer: a 1: 2 matched-pair analysis. Am. J. Clin. Oncol. 41(12), 1252-1256 (2018).

- This case-control study observes that $B R C A$-mutated breast cancer patients are more likely to develop brain metastases in a short time interval compared with wild-type BRCA controls matched for age, stage, ER expression and HER2 expression.

20. Azim HA, Abdel-Malek R, Kassem L. Predicting brain metastasis in breast cancer patients: stage versus biology. Clin. Breast Cancer. 18(2), e187-e195 (2018).

21. Pearce N. Analysis of matched case-control studies. BMJ 352, i969 (2016).

22. Hess KR, Esteva FJ. Effect of HER2 status on distant recurrence in early stage breast cancer. Breast Cancer Res. Treat. 137(2), 449-455 (2013).

23. Park H, Chang SK, Kim JY, Lee BM, Shin HS. Risk factors for distant metastasis as a primary site of treatment failure in early-stage breast cancer. Chonnam Med. J. 50(3), 96-101 (2014).

24. Laakmann E, Witzel I, Fasching PA et al. Development of central nervous system metastases as a first site of metastatic disease in breast cancer patients treated in the neoadjuvant trials GeparQuinto and GeparSixto. Breast Cancer Res. 21(1), 60 (2019).

25. von Minckwitz G, Huang C-S, Mano MS et al. Trastuzumab emtansine for residual invasive HER2-positive breast cancer. N. Engl. J. Med. 380(7), 617-628 (2018).

26. Morris PG, Murphy CG, Mallam D et al. Limited overall survival in patients with brain metastases from triple negative breast cancer. Breast J. 18(4), 345-350 (2012). 